Gut immunology

\title{
From hyperplasia to T cell lymphoma
}

N Cerf-Bensussan, N Brousse, C Cellier

\section{Disturbances in intraepithelial lymphocyte homeostasis in coeliac disease may lead to the emergence of lymphoid malignancies}

E nteropathy-type intestinal $\mathrm{T}$ cell lymphomas (EITCL) are a recognised complication of coeliac disease (CD). ${ }^{1}$ A recent survey confirmed that non-Hodgkin lymphomas, although rare, are the main cause of mortality in $\mathrm{CD}^{2}$ The mechanisms favouring the development of EITCL in CD patients but not in other chronic inflammatory bowel diseases remain elusive, but mounting evidence points to a profound disturbance in intraepithelial lymphocyte (IEL) homeostasis, leading to the emergence of lymphoid malignancies. A link between IELs and EITCL was first advocated in 1988 by Spencer et al who observed that most EITCL expressed the CD103 IEL marker. ${ }^{3}$ Two complementary observations suggested that EITCL derive from a reactive $\mathrm{T}$ cell population present in the intestine of CD patients: thus the same $\mathrm{T}$ cell clonal rearrangement was detected by Murray et al in EITCL and in the adjacent non-tumoral flat mucosa, ${ }^{4}$ and by Ashton-Key et al in non-lymphomatous ulcers of ulcerative jejunitis and in lymphomas, which later developed in these patients. ${ }^{5}$ Recent work in refractory sprue (RS) provided a missing link between IELs and lymphomas in CD.

RS is a coeliac-like enteropathy, primary or secondary resistant to a strict gluten free diet (GFD). Several conditions underlie villous atrophy resistant to GFD $^{6}$ (Cellier et al, in preparation) but the majority of RS complicate $\mathrm{CD}$ and are associated with massive expansion of IELs with normal cytology but clonal $\mathrm{T}$ cell receptor $\gamma$ (TCR $\gamma$ ) rearrangements and abnormal phenotype. ${ }^{78}$ The malignant nature of IELs in RS was demonstrated by the frequent association of RS with ulcerative jejunitis (30\%), and the outset of EITCL sharing the same clonality and phenotype after several months or years in approximately $20 \%$ of cases. $^{7910}$ RS can thus be regarded as a "cryptic or low grade T cell lymphoma" derived from IELs, and draws a link between IEL hyperplasia, characteristic of CD, and EITCL. In some CD patients however, EITCL develop directly without this first intermediary step. ${ }^{6}$

The report of seven new patients by Farstad and colleagues ${ }^{11}$ in this issue of
Gut, four with RS without overt high grade lymphomas and three with EITCL associated with TCR $\gamma$ clonal rearrangement $(3 / 3)$ and phenotypically abnormal IELs $(1 / 3)$ away from the tumour, concurs with previous work in RS and EITCL, and highlights two novel findings [see page 372]. One concerns the phenotype of IELs. Cellier et al initially reported that IELs in RS contained intracellular $\mathrm{CD} 3 \epsilon+$ but did not express surface $\mathrm{CD} 3 \epsilon, \mathrm{CD} 8$, or TCR. ${ }^{8}$ Herein, in $2 / 4$ cases of RS, IELs expressed a TCR $\beta$ chain. These results are in keeping with previous reports, ${ }^{69}$ and our unpublished results, showing TCR $\beta$ chain expression in $2 / 20$ patients. Interestingly, flow cytometry in one patient studied herein as in our two cases, showed that TCR $\beta$ chains remain exclusively intracellular. Farstad et al also suggest that the lack of CD8 is not a constant feature of abnormal IELs in RS and EITCL. ${ }^{11}$ Using flow cytometry, we detected weak CD8 expression in a fraction of abnormal IELs in 30\% of RS patients but only one and two of 20 cases were positive for CD8 by immunohistochemistry on fixed and frozen tissue sections, respectively ${ }^{7}$ (unpublished data). Daum et al in a recent report observed one case of CD8+ (CD56+) EITCL with CD8 + clonal IELs away from the tumour but concluded that clonality in EITCL and RS is generally associated with loss of CD8 and/or $\beta \mathrm{F} 1$ expression. ${ }^{6}$ Taken together, these data indicate that the phenotype of IELs in RS is very similar to that reported in EITCL, with the majority of cases being CD3+ $\beta$ F $1-C D 8-C D 4-$, but with some cases being CD8+ or $\beta \mathrm{Fl}+$; rare cases are positive for both markers. ${ }^{4}{ }^{6}$ These results emphasise the need to combine phenotype and molecular biology studies to investigate patients suspected of having RS or EITCL. They also support the idea that RS and EITCL share a common origin from IELs deprived of surface CD3-TCR complexes by a mechanism to be deciphered.

A second issue raised by Farstad and colleagues $^{11}$ concerns the predictive value of CD30 expression. The outcome of RS is variable and not easily predictable. A small number of patients respond to therapy, mainly corticoids and parenteral nutrition, and can be maintained symptom free for years on a GFD in spite of persistent monoclonal IELs, as illustrated in patient Nos 1 and 2 described by Farstad et al. In contrast, some patients develop high grade lymphomas while in others abnormal IELs do not form tumours but disseminate to lamina propria, blood, and eventually to other organs. Furthermore, some patients who do not develop lymphomas, rapidly die from untractable malabsorption. In the two latter cases, classical chemotherapy is ineffective or even deleterious because the abnormal lymphocytes divide too slowly to be efficiently destroyed by drugs interfering with cell proliferation. Discovery of prognosis markers would thus be useful to adjust therapy.

Farstad et al suggest that CD30 allows the early detection of overt lymphoma. ${ }^{11}$ Firstly, they detected some CD30+ blastlike IELs and lamina propria cells away from the tumours in their three cases of EITCL. Secondly, in one patient with RS without overt lymphoma, the rapidly fatal outcome was associated with the presence of $25 \%$ CD $30+$ blast-like IELs. No CD30+ IELs were however observed in another RS patient with rapid severe outcome, whereas a third patient with some CD30+ IELs was improved by parenteral nutrition and a GFD. Finally, the patient with many CD30+ IELs did not benefit from one attempted cure of CHOP. ${ }^{11}$ These observations illustrate the difficulties in predicting outcome and in propounding an appropriate treatment in RS patients. Apart from CD30, other immunohistochemical markers may help to detect transformation from low to high grade proliferation, such as proliferation markers or $\mathrm{p} 53$ detected by Murray et al on small lymphocytes in the bowel adjacent to EITCL. ${ }^{4}$ Nevertheless, as in patient No 3 reported by Farstad et al, none of these markers may be useful in predicting untractable malabsorption in the absence of overt lymphoma. Functional analysis of abnormal IELs in RS may identify criteria predictive of their aggressiveness for the mucosa or new targets for therapy, a pressing need given the lack of current efficient treatment for severely sick RS patients ${ }^{7}$ and the poor prognosis of EITCL. ${ }^{1}$ Insight into the mechanism(s) disturbing IEL homeostasis in CD may help to decipher the links between inflammation and lymphoid malignancies and to design treatments able to prevent or cure these rare but most severe complications of CD.

Gut 2002;51:304-305

\section{Authors' affiliations}

N Cerf-Bensussan, INSERM EPI-9925, Faculté

Necker-Enfants Malades, Paris, France N Brousse, INSERM EPI-9925, Faculté Necker-Enfants Malades, and Department of Pathology, Hôpital Necker-Enfants Malades, Paris, France 
C Cellier, INSERM EPI-9925, Faculté Necker-Enfants Malades, and Department of Gastroenterology, Hôpital Européen Georges Pompidou, Paris, France

Correspondence to: N Cerf-Bensussan, INSERM EPI-9925, Faculté Necker-Enfants Malades, 156 Rue de Vaugirard, 75737 Paris Cedex 15,

France; cerf@necker.fr.

\section{REFERENCES}

1 Gale J, Simmonds PD, Mead GM, et al. Enteropathy-type intestinal T-cell lymphoma: clinical features and treatment of 31 patients in a single center. J Clin Oncol 2000;18:795-803.

2 Corrao G, Corazza GR, Bagnardi V, et al. Mortality in patients with coeliac disease and their relatives: a cohort study. Lancet 2001;358:356-61
3 Spencer J, Cerf-Bensussan N, Jarry A, et al Enteropathy-associated T cell lymphoma (malignant histiocytosis of the intestine) is recognized by a monoclonal antibody (HML-1) that defines a membrane molecule on human mucosal lymphocytes. Am J Pathol 1988:132:1-5.

4 Murray A, Cuevas EC, Jones DB, et al. Study of the immunohistochemistry and $T$ cell clonality of enteropathy-associated T cell lymphoma. Am J Pathol 1995:146:509-19.

5 Ashton-Key M, Diss T, Pan L, et al. Molecular analysis of T-cell clonality in ulcerative jejunitis and enteropathy-associated T-cell lymphoma. Am J Pathol 1997;151:493-8.

6 Daum S, Weiss D, Hummel $M$, et al. Frequency of clonal intraepithelial $\mathrm{T}$ lymphocyte proliferations in enteropathy-type intestinal T cell lymphoma, coeliac disease, and refractory sprue. Gut 2001;49:804-12.

7 Cellier C, Delabesse E, Helmer, et al. Refractory sprue, coeliac disease, and enteropathy-associated T-cell lymphoma. French Coeliac Disease Study Group. Lancet 2000;356:203-8.

8 Cellier C, Patey N, Mauvieux L, et al. Abnormal intestinal intraepithelial lymphocytes in refractory sprue. Gastroenterology 1998;114:471-81

9 Carbonnel F, Grollet-Bioul L, Brovet J, et al. Are complicated forms of celiac disease cryptic T-cell lymphomas? Blood 1998;92 3879-86.

10 Badgi E, Diss T, Munson P, et al. Mucosal intraepithelial lymphocytes in enteropathy-associated T-cell lymphoma, ulcerative jejunitis, and refractory celiac sprue constitute a neoplastic population. Blood 1999;94:260-4.

11 Farstad I, Johansen F-E, Vlatkovic L, et al. Heterogeneity of intraepithelial lymphocytes in refractory sprue: potential implications of CD30 expression. Gut 2002;51:372-8.

\section{Infection and irritability}

\section{R Lea, P J Whorwell}

\section{A proportion of patients with irritable bowel syndrome report an apparent association between the onset of symptoms and a dysenteric illness}

C haudhary and Truelove were amongst the first to recognise that a proportion of patients with irritable bowel syndrome (IBS) report an apparent association between the onset of symptoms and a dysenteric illness. The concept of "post-dysenteric IBS" (PD-IBS) has now been widely accepted with claims suggesting that it accounts for anything up to $25 \%$ of the totality of the condition. However, careful questioning of these patients sometimes, but not always, suggests that they may have had a "forme fruste" of the disorder before their infection, raising the possibility that there may be two forms of the illness, one being an exacerbation of a pre-existing disorder and another where the condition appears to arise de novo. Ultimately, issues surrounding the natural history of PD-IBS will only be resolved by detailed prospective and retrospective studies, such as the one reported by Neal and colleagues ${ }^{2}$ in this issue of Gut [ see page 410]

Given that dysentery leads to irritable bowel symptoms, what mechanisms might be responsible? Do the dysenteric organisms cause the problem directly, and if so, are some more noxious than others? Alternatively, does the resulting diarrhoea lead to a form of non-specific sensitisation?
A retrospective analysis of the prevalence of gastrointestinal symptoms following microbiologically confirmed gastroenteritis suggested that there was no difference in the risk of IBS between bacterial species. ${ }^{3}$ However, it is noteworthy that up to half of the stool cultures obtained in some studies of PD-IBS may in fact be negative. This suggests that viruses or possibly other pathogens such as parasites may also be capable of provoking IBS in some individuals. There has been relatively little work addressing the possible role of viruses in IBS although it is tempting to speculate that such agents might be implicated in some cases, especially as symptoms of IBS are very common in chronic fatigue syndrome for which a viral aetiology has been proposed

In addition to the role of different pathogens, it is possible that exposure to other factors which may be associated with the infection could be critical to the subsequent development of IBS. For instance, it has been shown that diarrhoea induced by polyethylene glycol can lead to rectal sensitisation, ${ }^{4}$ suggesting that diarrhoea per se is important. It is also well known that the use of antibiotics often results in diarrhoea, and there is evidence that patients suffering from dysentery treated with antibiotics are more likely to develop IBS compared with those who do not receive this form of therapy. Furthermore, this effect has been observed with antibiotics used for reasons other than dysentery. ${ }^{5}$ A further explanation for the deleterious effect of antibiotics in IBS might be alteration of the bacterial flora in the gut. If this is the case, one might predict that measures aimed at favourably modifying the gut flora might offer therapeutic promise, and reports of the beneficial effect of probiotics would seem to support this view.

Gastroenteritis can lead to disruption of the gastrointestinal mucosa resulting in excessive antigenic exposure and loss of the brush border. However, it is well known that the resultant disaccharidase deficiency is usually transient, and therefore unlikely to be contributory to the pathogenesis of PD-IBS. A similar mechanism may lead to bile salt malabsorption, and a group of patients with chronic gastrointestinal symptoms and documented bile salt malabsorption have been reported as relating the onset of their problems to an episode of gastroenteritis. Bile salt chelating therapy has been used in this particular situation with apparent success, although it is generally an unrewarding form of treatment for IBS in general. Food intolerance has also been reported as being important in some patients with IBS, but there is little evidence that an immune mediated process due to increased antigen exposure is involved. However, abnormal bacterial fermentation, a process capable of elaborating toxic short chain fatty acids, has been suggested as a possible cause of food intolerance, and may follow both gastroenteritis and antibiotic usage.

Psychosocial factors are known to be important in IBS. Several studies, including that of Neal and colleagues, ${ }^{2}$ which specifically relate to PD-IBS have shown that the risk of developing persistent symptoms following dysentery is related to the presence of psychopathology. Although gastroenteritis may lead to 
physiological changes that predispose to IBS, there is evidence that an adverse psychosocial milieu is necessary for the condition to fully develop. ${ }^{6}$ This is perhaps not surprising as it is now well recognised that stress can affect the immune, and hence the inflammatory response. ${ }^{7}$ Similarly, stress may increase intestinal permeability, an observation that may be particularly relevant as increased gut permeability has been demonstrated in some patients with PD-IBS. ${ }^{8}$

It would seem reasonable to assume that whatever the triggering factor, an inherited predisposition for IBS might be necessary. This is suggested by the observation that IBS tends to cluster within families, although this could also be explained by environmental factors and indeed, similarities in health related behaviour have been observed between close relatives of those with IBS. Nevertheless, twin studies have shown an increased prevalence of IBS in mono compared with dizygotic twins, ${ }^{9}{ }^{10}$ which might support a genetic background, but a study involving mono and dizygotic twins separated at birth would be required in order to reach a firm conclusion. Laboratory evidence also provides some support for the concept that inheritance is an important factor in the development of IBS. Studies on cytokines, which are known to be involved in the modulation of intestinal inflammation, have shown that mice lacking the interleukin 10 gene develop a spontaneous form of chronic enterocolitis, and that patients with ulcerative colitis are more likely to have genotypes associated with a lower production of interleukin 10. Similarly, a significantly reduced prevalence of the "high producer" gene for interleukin 10 has been reported in a group of unselected patients with IBS. ${ }^{11}$

It is almost 40 years since Chaudhary and Truelove wrote their classic paper identifying the PD-IBS subgroup. We now know that female sex, younger age, prolonged duration of the initial illness, and psychological comorbidity appear to be important risk factors, and that sufferers usually have the diarrhoea predominant form of the condition. However, there is still much to learn, and emerging technologies will undoubtedly aid this process.

\section{Gut 2002;51:305-306}

\section{Authors' affiliations}

R Lea, P J Whorwell, Academic Department of Medicine, University Hospital of South Manchester, Manchester, UK

Correspondence to: Dr P J Whorwell, Academic Department of Medicine, Education and Research Centre, Wythenshawe Hospital, Southmoor Road, Wythenshawe, Manchester M23 9LT, UK

peter.whorwell@smuht.nwest.nhs.uk

\section{REFERENCES}

1 Chaudhary NA, Truelove SC. The irritable bowel syndrome. Q J Med 1962;31:307-22.
2 Neal KR, Barker L, Spiller RC. Prognosis in post-infective irritable bowel syndrome: a six year follow up study. Gut 2002;51:000-0.

3 Neal KR, Hebden J, Spiller R. Prevalence of gastrointestinal symptoms six months after bacterial gastroenteritis and risk factors for development of the irritable bowel syndrome: postal survey of patients. BM 1997;314:779-82

4 Houghton LA, Wych J, Whorwell PJ. Acute diarrhoea induces rectal sensitivity in women but not men. Gut 1995;37:270-3.

5 Maxwell PR, Rink E, Kumar D, et al. Antibiotics increase functional abdominal symptoms. Am J Gastroenterol 2002;97:104-8.

6 Gwee K-A, Leong Y-L, Graham C, et al. The role of psychological and biological factors in postinfective gut dysfunction. Gut 1999;44:400-6.

7 Collins SM. Stress and the gastrointestinal tract IV. Modulation of intestinal inflammation by stress: basic mechanisms and clinical relevance. Am J Physiol Gastrointest Liver Physiol 2001;280:G315-18.

8 Spiller RC, Jenkins D, Thornley JP, et al. Increased rectal mucosal enteroendocrine cells, T lymphocytes, and increased gut permeability following acute Campylobacter enteritis and in post-dysenteric irritable bowel syndrome. Gut 2000;47:804-11.

9 Levy RL, Jones KR, Whitehead WE, et al. Irritable bowel syndrome in twins: Heredity and social learning both contribute to etiology. Gastroenterology 2001;121:799-804.

10 Morris-Yates A, Talley NJ, Boyce PM, et al. Evidence of a genetic contribution to functional bowel disorder. Am J Gastroenterol 1998:93:1311-17.

11 Cheng J, Gonsalkorale M, Perry C, et al. IL- 10 and TGF- $\beta$ genotype in irritable bowel syndrome: evidence to support an inflammatory component? Gastroenterology 2000;118(suppl 1):A184

\section{Top down or bottom up? Competing management structures in the morphogenesis of colorectal neoplasms}

\section{N A Wright, R Poulsom}

Modifier genes may influence the severity, or adenoma number, of familial adenomatous polyposis in humans through tumour initiation rather than progression

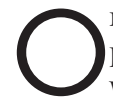
ne of the earliest tumour suppressor genes to be identified was APC. Germline mutations in APC are found in familial adenomatous polyposis (FAP) and second hits lead to the development of often many hundreds of adenomas in the colon and rectum, some of which progress to cancer if untreated. Many sporadic adenomas, and their ensuing carcinomas, show APC mutations, and FAP remains an important paradigm for the commoner sporadic form. Thus recent studies from the Tomlinson laboratory ${ }^{1}$ show a very close linear relationship between the macroscopic-or naked eye-count of adenomas in excised FAP colons and the count made microscopically from adenomas occupying one crypt (the unicryptal or monocryptal adenoma, fig 1) upwards. Such a close relationship strongly indicates that progression from microadenomas to macroscopic size is essentially random, that variation in disease severity (number of adenomas) results from differences in the number of microadenomas rather than disease progression, and importantly, that the selective advantages provided by different APC mutations act on tumour initiation rather than progression. A paper in this issue of Gut, also from the Tomlinson laboratory, ${ }^{2}$ analyses the effects of putative modifier genes: the severity of the disease was related to the site of the mutation, as might be expected, but first degree relatives showed polyp counts which were more similar than more distant relatives [see page 420]. These observations indicate that modifier genes influence the severity of FAP, again through tumour initiation. Furthermore, the finding of a constant microadenoma density as the colon is traversed ${ }^{1}$ suggests that initiation of FAP adenomas 


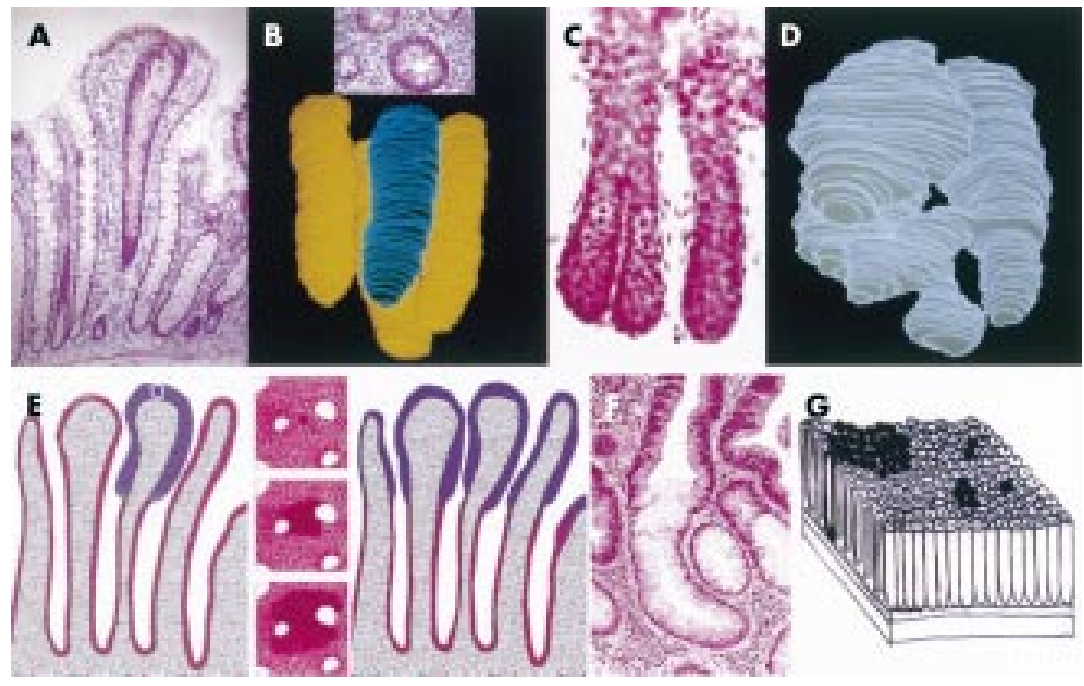

Figure 1 (A) A monocryptal or unicryptal adenoma. (B) A three dimensional reconstruction of a unicryptal adenoma (inset) from serial sections, showing the adenoma in blue. Note that the adenomatous epithelium extends to the base of the crypt. (C) The mechanism of crypt fission in the normal colon whereby a crypt divides into two by this fission process. (D) A larger adenoma showing expansion by basal fission and budding. (E) Lateral migration at the margins of an adenoma, with adenomatous epithelium invading crypt territories (reproduced with permission from Shih and colleauges, ${ }^{4}$ copyright 2001 National Academy of Sciences, USA). (F) "Top down models" of adenoma morphogenesis where either a single cell incurs APC inactivation, passes to the top of the crypt and proliferates, or transforms in situ at the top of the crypt. Both concepts lead to expansion of the clone in the intercrypt zone (from Shih and colleauges ${ }^{4}$ ). (G) How mutated clones expand in the colorectal epithelium by crypt fission.

are spontaneous events rather than environmentally produced, which of course has considerable potential implications for sporadic adenomas.

These observations underline the pivotal early events in colonic carcinogenesis: establishment of the mutant clone, its evolution to a microadenoma, and its development into a tumour recognisable by the naked eye. The molecular events associated with these stages are clear: in FAP a second hit in the APC gene is sufficient to give microadenoma development. ${ }^{3}$ But a further recent article from the Vogelstein laboratory ${ }^{4}$ has drawn on some earlier morphological studies to challenge contemporary concepts of how such mutant cells establish themselves and develop into an adenoma. Struck by the appearances in some early non-FAP adenomas (fig 1), dysplastic cells were seen only at the orifices and luminal surface of colonic crypts. Shih et al determined loss of heterozygosity ( $\mathrm{LOH}$ ) for APC, and nucleotide sequence analysis of the mutation cluster region of the APC gene was applied to microdissected well orientated histological sections of these adenomas. Not surprisingly perhaps, half the sample showed LOH in the upper portion of the crypts and most of these had a truncating APC mutation. Those cases without LOH showed a truncating mutation, again confined to the dysplastic epithelium at the crypt apex. Moreover, these cells showed intense proliferative activity, with nuclear localisation of $\beta$-catenin, supporting the presence of an
APC mutation in these apical dysplastic cells. Several earlier morphological studies have drawn attention to the same appearances, ${ }^{5-9}$ including those in FAP. ${ }^{9}$

This morphological and immunohistochemical profile was apparently virtually always present in nearly every crypt of each adenoma studied. Two models for adenoma morphogenesis were proposed (fig 1): in the first, mutant cells appear in the intracryptal zone between crypt orifices and, as the clone expands, cells migrate laterally and downwards to displace the normal epithelium of adjacent crypts. Alternatively, a mutant cell in the crypt base, classically the site of the stem cell compartment, ${ }^{10}$ migrates to the crypt apex where it expands as before (fig l).

This "top down morphogenesis" has profound implications for concepts of stem cell biology in the gut. Most evidence indicates that crypt stem cells are found at the origin of the cell flux, near the crypt base. ${ }^{11}$ Their repertoire includes all crypt cell lineages, metaplastic and reparative cell lineages, the genesis of new crypts and, as is widely believed, gastrointestinal tumours. ${ }^{12}$ These proposals by Shih and colleagues ${ }^{4}$ either re-establish the stem cell compartment in the intracryptal zone or make the intracryptal zone a locus where stem cells, having acquired a second hit, clonally expand.

Where the concepts of Crabtree and colleagues $^{12}$ and Shih and colleagues ${ }^{4}$ diverge is in the recognition of the earliest lesion, the unicryptal adenoma, where the dysplastic epithelium occupies an entire single crypt. ${ }^{1}$ These lesions are very common in $\mathrm{FAP}^{13}$ and while rare in non-FAP patients, have certainly been described.$^{14}$ Here, a stem cell apparently acquires a second hit and expandseither stochastically or more probably because of a selective advantage to colonise the entire crypt. Such lesions are thus clonal. ${ }^{13}$ Similar crypt restricted expansion has been well documented in mice after ENU treatment, ${ }^{15}$ and also in humans heterozygous for the OAT $(O$ acetyl transferase) gene where, after $\mathrm{LOH}$, initially half and then the whole crypt is colonised by the progeny of the mutant stem cell. ${ }^{16}$ Interestingly, OAT+/ OAT- individuals with FAP show increased rates of stem cell mutation with clustering of mutated crypts. ${ }^{16}$

In this scenario, in sharp contrast, the mutated clone further expands, not by lateral migration but by crypt fission, where the crypt divides, usually symmetrically at the base, or by budding (fig 1). In several studies, fission of adenomatous crypts is regarded as the main mode of adenoma progression, certainly in FAP where such events are readily evaluated, ${ }^{17}{ }^{18}$ but also in sporadic adenomas. $^{19}$ In fact, the nonadenomatous mucosa in FAP, with only one APC mutation, shows a large increase in the incidence of crypts in fission. ${ }^{17}$ Aberrant crypt foci, lesions which are putative precursors of adenomas, which can show k-ras and APC mutations, ${ }^{20}$ grow by crypt fission ${ }^{2122}$ as do hyperplastic polyps. ${ }^{23}$ But this concept does not exclude the possibility that the clone also expands by lateral migration and downward spread into adjacent crypts; this model of morphogenesis is conceptually quite different from that proposed by Shih and colleagues. ${ }^{4}$

Finally, there are other reasons for finding crypts containing a mixture of mutant and wild-type cells-or APC-/and $A P C+/-$ cells. Bjerkes and colleagues $^{24}$ found crypts harbouring cells staining both positively and negatively for APC protein in FAP although these were not spatially distinct, and were construed as crypts containing at least two stem cell lineages. Moreover, at the margins of FAP adenomas, serial section reconstruction has shown normal crypts in continuity with two or three adenomatous crypts, ${ }^{17}$ interpreted as adjacent normal crypts transforming into adenomatous crypts. Since crypts are clonal units, ${ }^{13}$ this would explain the observation that some $75 \%$ of microadenomas in an FAP patient and in Min mice appear polyclonal. ${ }^{1325}$

The concept that the severity of the disease, or adenoma number, depends on initiation rather than progression, ${ }^{12}$ brings these early events into sharp focus. The debate also extends into how clonal patches of dysplasia spread in the 
colon in ulcerative colitis ${ }^{26}$ - "top down" by lateral migration or "bottom up" by crypt fission, or both? Which management structure prevails will have considerable implications for gut biology.

Gut 2002;51:306-308

\section{Authors' affiliations}

N A Wright, R Poulsom, Histopathology Unit, Cancer Research UK, 44 Lincoln's Inn Fields, London WC2A 3PX, UK, and Department of Histopathology, Bart's and the London, Queen Mary's School of Medicine and Dentistry, University of London, London, UK

Correspondence to: Professor N A Wright, Histopathology Unit, Cancer Research UK, 44 Lincoln's Inn Fields, London WC2A 3PX, UK warden@qmul.ac.uk

\section{REFERENCES}

1 Crabtree MD, Tomlinson IPM, Talbot IC, et al. Variation in the severity of colonic polyposis in FAP results from differences in tumour initiation rather than progression and depends relatively little on patient age. Gut 2001:49:540-3.

2 Crabtree MD, Tomlinson IPM, Hogson, SV, et al. Explaining vartiation in familial adenomatous polyposis: relationship between genotype and phenotype and evidence for modifier genes. Gut 2002:51:420-3.

3 Lamlum $\mathbf{H}$, llyas $M$, Wheeler J, et al. APC mutations in sporadic colorectal tumors: a mutational "hotspot" and interdependence of the "two hits". Proc Natl Acad Sci USA 2000:97:2225-8.

4 Shih IM, Wang TL, Traverso G, et al. Top-down morphogenesis of colorectal tumors. Proc Natl Acad Sci USA 2001;98:2640-5.
5 Cole JW. The adenoma-carcinoma sequence in the experimental animal. Major Probl Pathol 1978;10:119-25.

6 Maskens AP. Histogenesis of adenomatous polyps in the human large intestine. Gastroenterology 1979:77:1245-51.

7 Nakamura S, Kino L. Morphogenesis of minute adenomas in familial polyposis coli. $J$ Natl Cancer Inst 1984;73:41-9.

8 Polyak K, Hamilton SR, Vogelstein B, et al Early alteration of cell-cycle-regulated gene expression in colorectal neoplasia. Am Pathol 1996:148:381-7.

9 Lane N, Lev R. Observations on the origin of adenomatous epithelium of the colon: serial section studies of minute polyps in familial polyposis. Cancer 1965;16:751-64.

10 Bjerknes $\mathbf{M}$, Cheng $\mathrm{H}$. The stem-cell zone of the small intestinal epithelium. I. Evidence from Paneth cells in the adult mouse. Am J Anat 1981;160:51-63.

11 Bjerknes M, Cheng H. Clonal analysis of mouse intestinal epithelial progenitors. Gastroenterology 1999;116:7-14.

12 Wright NA. Epithelial stem cell repertoire in the gut: clues to the origin of cell lineages, proliferative units and cancer. Int J Exp Pathol 2000;81:117-43.

13 Novelli MR, Williamson JA, Tomlinson IP, ef al. Polyclonal origin of colonic adenomas in an $\mathrm{XO} / \mathrm{XY}$ patient with FAP. Science 1996;272:1187-90.

14 Woda BA, Forde K, Lane N. A unicryptal colonic adenoma, the smallest colonic neoplasm yet observed in a non-polyposis individual. Am J Clin Patho 1977;68:631-32.

15 Park HS, Goodlad RA, Wright NA. Crypt fission in the small intestine and colon. A mechanism for the emergence of G6PD locus-mutated crypts after treatment with mutagens. Am J Pathol 1995; 147: 1416-27.

16 Campbell F, Geraghty JM, Appleton MA, et al. Increased stem cell somatic mutation in the non-neoplastic colorectal mucosa of patients with familial adenomatous polyposis Human Pathol 1998;29: 1532-5.
17 Wasan HS, Park HS, Liu KC, et al. APC in the regulation of intestinal crypt fission. J Pathol 1998;185:246-55.

18 Chang WW, Whitener CJ. Histogenesis of tubular adenomas in hereditary colonic adenomatous polyposis. Arch Pathol Lab Med 1989;113:1042-9

19 Wong WM, Mandir N, Goodlad RA, et al. Histogenesis of human colorectal adenomas and hyperplastic polyps: the role of cell proliferation and crypt fission. Gut 2002;50:212-7.

20 Smith AJ, Stern HS, Penner M, et al. Somatic $A P C$ and $k$.ras codon 12 mutations in aberrant crypt foci from human colons. Cancer Res 1994;54:5527-30.

21 Fujimitsu $\mathbf{Y}$, Nakanishi $\mathrm{H}$, Inada $\mathrm{K}$, et al. Development of aberrant crypt foci involves a fission mechanism as revealed by isolation of aberrant crypts. Jpn J Cancer Res 1996:87: 1199-203.

22 Siu IM, Robinson DR, Schwartz S, et al. The identification of monoclonality in human aberrant crypt foci. Cancer Res 1999;59:63-6.

23 Oohara T, Ogino A Tohma H. Histogenesis of microscopic adenoma and hyperplastic (metaplastic) gland in nonpolyposis coli. Dis Colon Rectum 1981;24:375-84.

24 Bierkes $\mathbf{M}$, Cheng $\mathrm{H}$, Kim H, et al. Clonality of dysplastic epithelium in colorectal adenomas from familial adenomatous polyposis patients. Cancer Res 1997;57:355-61.

25 Merritt AJ, Gould KA, Dove WF. Polyclonal structure of intestinal adenomas in ApcMin/+ mice with concomitant loss of Apc+ from all tumor lineages. Proc Natl Acad Sci USA 1997; 84:13927-31

26 Lyda MH, Noffsinger A, Belli J, et al. Multifocal neoplasia involving the colon and appendix in ulcerative colitis: pathological and molecular features. Gastroenterology 1998; 115: 1566-73. 